\title{
Diaphonization: A Recipe to Study Teeth
}

\author{
${ }^{1}$ Kiran Rehman, ${ }^{2}$ Farhan Raza Khan, ${ }^{3}$ Sadia Habib
}

\section{ABSTRACT}

Aim: There are various techniques to study root canal morphology and diaphonization is one of them. There are various methods of decalcification and diaphonization, cited in literature and the main aim of this paper was to give a brief account of the various techniques and share our experience of the technique at a teaching institution in Karachi, Pakistan.

Materials and methods: Diaphonization is one of the oldest methods and is based on decalcification of teeth followed by clearing and dye penetration. The specimen is later studied under microscope without sectioning.

Results: After the process of clearing a three-dimensional (3D) structure of the internal canal anatomy was visible with naked eye.

Conclusion: This paper entails a detailed historical background as well as the author's technique including percentages of various chemicals used and the timing of immersion of teeth into these agents.

Clinical significance: The read out is simple and can be subjected to interpretation by direct observation under microscope and can be helpful for students undertaking research in not only the discipline of dentistry but also in other fields such as botany and zoology.

Keywords: Diaphonization, Clearing technique, Root canal morphology, Vertucci classification.

How to cite this article: Rehman K, Khan FR, Habib S. Diaphonization: A Recipe to Study Teeth. J Contemp Dent Pract 2015;16(3):248-251.

\section{Source of support: Nil}

Conflict of interest: None

\section{INTRODUCTION}

The variation in the root canal anatomy has been a widely studied subject from the early 1800s. The first detailed and comprehensive description of the root canal anatomy

\footnotetext{
${ }^{1}$ Department of Operative Dentistry, Liaquat National Hospital and Medical College, Karachi, Pakistan

${ }^{2}$ Department of Operative Dentistry, Aga Khan University, Karachi, Pakistan

${ }^{3}$ Juma Research Laboratory, Aga Khan University, Karachi Pakistan

Corresponding Author: Kiran Rehman, Assistant Professor Department of Operative Dentistry, Liaquat National Hospital and Medical College, Karachi 74800, Pakistan Phone: +92 3212672957, e-mail: dr.k.rehman@gmail.com
}

was studied and published by Carabelli in 1842, who presented his work in the form of drawings of sectioned teeth. Carabelli not only studied the internal anatomy but also the external morphologies of all groups of teeth. ${ }^{1}$

In the past, various techniques have been used to study the canals in in vitro studies, such as

- Direct observation with the aid of a microscope

- Macroscopic sections

- Microscopic sections

- Transverse sections

- Micrometric measurements

- Examination of intraoral radiographs

- Dye penetration and clearing

- Filling and clearing

- Scanning electron microscopy

However, clearing technique has been to date the best tool to study internal tooth anatomy. It is a powerful educational tool as it gives a three-dimensional (3D) view of the pulp chambers and the root canals.

Various techniques have been used to clear teeth and a number of demineralizing and clearing agents have been proposed. ${ }^{2-6}$

\section{REVIEW}

Muhlreiter in 1870 was the first one after Carabelli to vigorously investigate the internal anatomies of teeth by sectioning them in all planes and described the internal anatomy in detail. He published his findings in his book Anatomie des Mundes. ${ }^{7}$ In 1890, GV Black also contributed to the subject of root canal anatomy in his first book 'Descriptive anatomy of the human teeth'. Preiswerck in 1901 injected molten metal into the pulp chamber followed by complete decalcification of the tooth and obtaining a 3D model of the canal anatomy.

In the years that followed, various materials were used to obtain 3D models of the root canal system, but the most impressive work was published by Hess et $\mathrm{al}^{9}$ in 1917. He injected the root canal system with vulcanized rubber and then removed it after decalcifying the tooth. His work was the first description of the high degree of variations seen in canal anatomies. Hess et al published numerous papers demonstrating a large number of pulpal vessels with intricate anatomies and varying curvatures, as well as few to several anastomoses and junctions.

However, many of the earlier studies proved to be inaccurate until 1965, when Rankine-Wilson and Henry 
published their report on the canal anatomy of mandibular anterior teeth. ${ }^{10}$

Further research began following the Rankine-Wilson and Henry's paper, and researchers started investigating the canal anatomies of other frequently endodontically treated teeth. Rankine-Wilson and Weine later investigated the mesiobuccal root of maxillary first molar and much research was done focusing on this particular root. Following this, Weine formulated a classification for root canal systems which is the most basic classification so far.

\section{Diaphonization of Teeth}

Injecting dye into the canal spaces and decalcifying the tooth is known as diaphonization. It was first done by Okumura ${ }^{11}$ who also classified the root canals in relation to their anatomical distribution.

This technique is more commonly used in bone histology studies and has been in use to study bone turnover rates in various diseases of bone. ${ }^{12}$ In dental research, clearing method is not only used to study internal anatomy but also in studies of micro leakage through various filling materials.

Diaphonization techniques have been used since the early 19 th century. Okumura performed in-depth studies of the pulp anatomy using clearing techniques followed by injecting dye into the canal system to make the canal anatomy obvious. Dedeus in 1960 was the first author to systematically study the root canal anatomy of all dental groups using clearing technique (diaphonization). ${ }^{13}$

Canals can be assessed by various methods, such as sectioning teeth, decalcification techniques, various radiographic techniques, such as standard periapical radiographs, computed tomography scans as well as cone beam computed tomography. Radiographic techniques are more useful for clinical settings. The pulpal soft tissue can only be assessed in decalcified sections which otherwise is lost in ground sections. In routine histopathology, decalcification of bone and teeth is often an essential and important step during tissue processing to examine and understand the internal anatomy of calcified tissues. The aim of decalcification is to remove calcium salts from mineralized tissue using chemical solutions like acids and chelating agents, while preserving the organic portions. Further research was carried out by Pomeranz and Fishelberg as well as Vertucci using acid to decalcify the teeth followed by dehydrating and injecting a dye to study the canal anatomy.

The basic steps of diaphonization include:

- Decalcification

- Dehydration

- Immersion in clearing agent
- Dye penetration

- Fixation or storage.

In the past researchers have used many different techniques and materials to decalcify and dehydrate teeth and various clearing and storage agents have been used.

The clearing technique as described by Robertson et $\mathrm{al}^{4}$ involves soaking teeth into 5\% nitric acid, for 72 hours, changing the acid every 24 hours, and stirring every 8 hours. The teeth are then dehydrated in ascending grades of 60 to $100 \%$ alcohol rinses and then finally rendered transparent after dipping in a clearing agent, such as methyl salicylate or xylene. This is the method used in the current study as well and is the most widely used and accepted method.

\section{Our Technique (developed and optimized by Investigators at Aga Khan University, Pakistan)}

Access cavities were prepared using a round bur (No. 2 round bur) and the canal orifices were observed with the naked eye. The teeth were stored in $10 \%$ formalin for 3 days. They were then washed in running water for 2 hours to remove all traces of formalin and sodium hypochlorite, after which they were transferred to $15 \mathrm{ml}$ bottles for the remainder of the study.

\section{Decalcification Procedure}

The teeth were each immersed in 5\% nitric acid for decalcification for 72 hours. The acid was changed every 24 hours and stirred once every 8 hours. The end-point of decalcification was determined by taking a radiograph of three sample teeth after 72 hours (Figs 1 to 3 ).

The teeth were then washed in running water for 2 hours after which they were dehydrated using ascending grades of isopropyl alcohol:

70\%: 3 hours
80\%: 3 hours
90\%: 2 hours

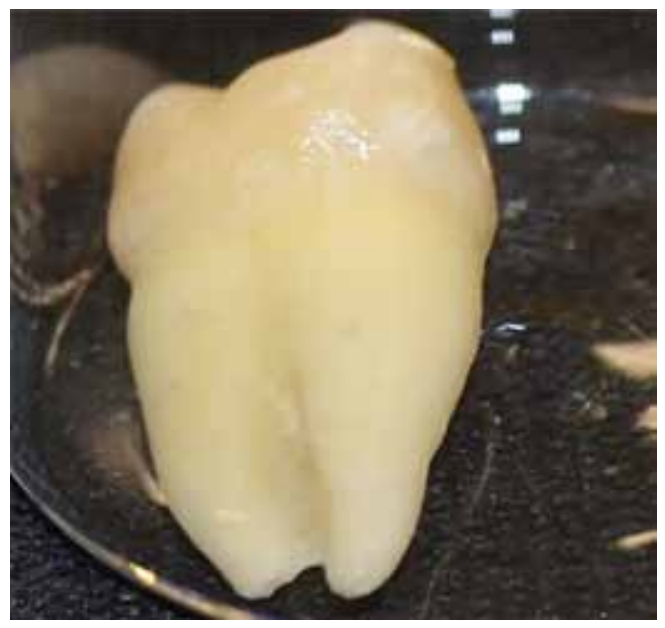

Fig. 1: Tooth before decalcification 

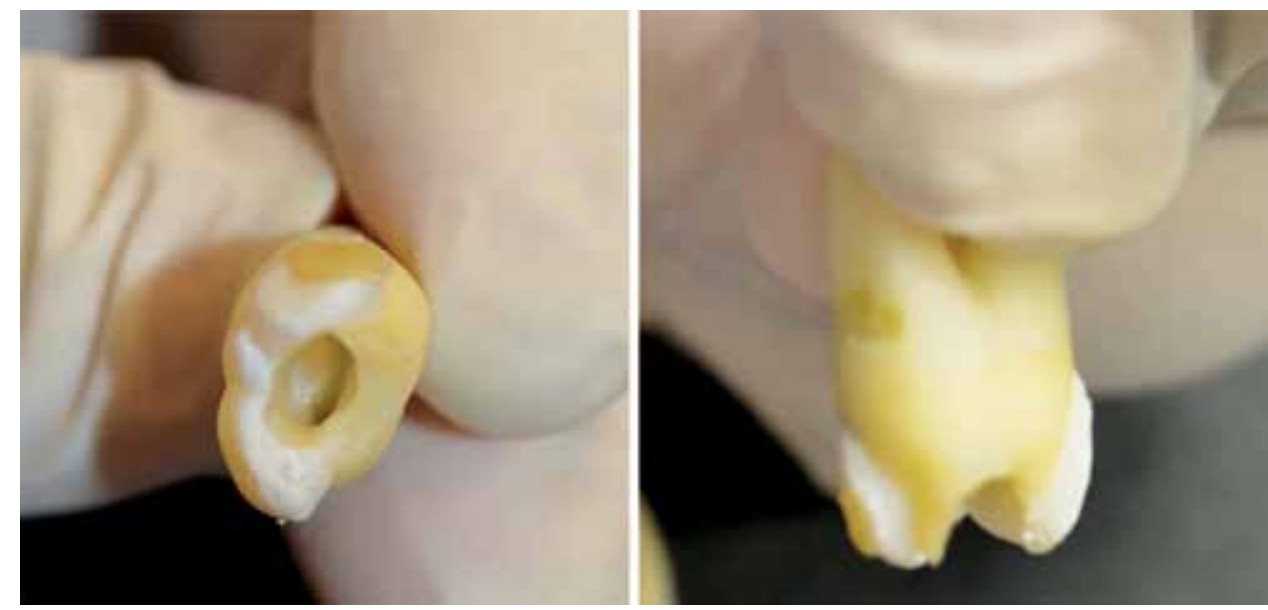

Fig. 2: Partially demineralized tooth after 24 hours

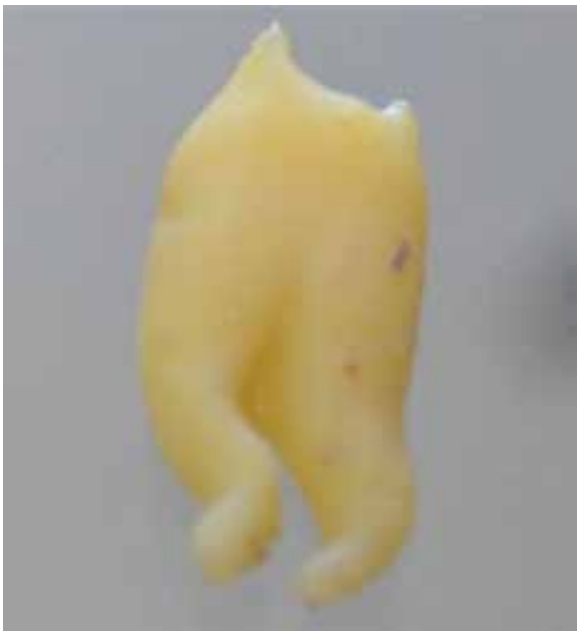

Fig. 3: Completely demineralized tooth after 72 hours of immersion in $5 \%$ nitric acid

Solution was refreshed at every hour.

100\%: 1 hour

Finally, the teeth were immersed in xylene to become transparent, and left in it for 2 days. India ink which is an oil based dye was then injected into the access cavity using an empty xylocaine $1.8 \mathrm{ml}$ cartridge, and a 32 gauge infiltration needle, inserted on a surgical plunger. The India ink was injected into the access cavity with the apex of the tooth toward a high volume suction to create a negative pressure that would cause the ink to flow from the coronal to the apical portion (Fig. 4).

The anatomy of the root canal was observed under light microscope at $2 \times$ magnification and classified based on Vertucci's classification.

The technique that was used in our study was first described by Robertson et al in $1980 .{ }^{4}$ Different researchers have modified the technique with different chemicals and using 5 to $11 \%$ nitric acid, ${ }^{14,15} 20 \%$ formic acid, ${ }^{16} 40 \%$ solution of ion exchange resin and formic acid ${ }^{17}$ or $5 \%$ hydrochloric acid. ${ }^{18}$

In this study, we used 5\% nitric acid diluted with distilled water, soaking the teeth in it for 72 hours. The

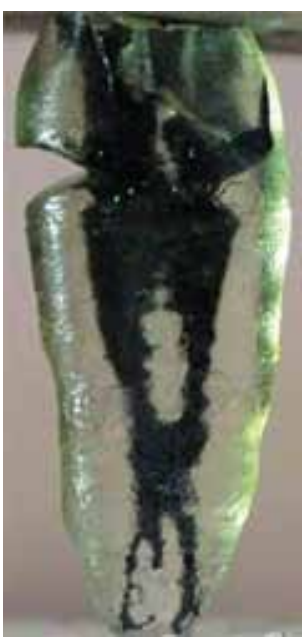

Fig. 4: Cleared tooth after dye penetration

sample bottles containing the teeth soaked in acid were vibrated every 8 hours using a plaster mixing vibrator (Silfradent VIB24) so that acid could be evenly distributed and the teeth are dipped in acid evenly. The surfaces facing the glass were slow to demineralize and therefore, it was important to keep all surfaces soaked for the same amount of time. It has been reported that shrinkage of organic tooth tissue could occur during the demineralization process, and that this phenomenon may be avoided if a weak concentration of acid is used. ${ }^{4}$ Alcohol-dip timing is one of the most important factors, after demineralization, which affects the outcome of the tooth transparency, therefore, the regimen that we followed was 70 and $80 \%$ alcohol for 3 hours, 90\% alcohol for 2 hours, changing the alcohol every hour, and $100 \%$ alcohol for 1 hour. The teeth after the $100 \%$ alcohol dip were then shifted to xylene to render them transparent and left in xylene for 2 days. After dye injection, methyl salicylate was then used to store the teeth for the remainder of the study. The only reason for storing them in methyl salicylate as opposed to xylene was because of the highly corrosive nature of xylene. 
In addition to dentistry, disciplines, such as botany and zoology also utilize this method to clear the specimens without sectioning them to understand their internal anatomy and morphological characteristics.

\section{CONCLUSION}

Diaphonization is an interesting method that can be employed by dental students, residents in graduate studies or fellowship trainees for their research work relating to internal tooth morphology. The read-out is simple and can be subjected to interpretation by direct observation under microscope. This paper entails a detailed historical background as well as the authors' technique including percentages of various chemicals used and the timing of immersion of teeth into these agents.

\section{REFERENCES}

1. Carabelli G, von Lunkaszprie E. Anatomie des Mundes: Braumüller und Seidel; 1842.

2. Neelakantan P, Subbarao C, Subbarao CV. Comparative evaluation of modified canal staining and clearing technique, conebeam computed tomography, peripheral quantitative computed tomography, spiral computed tomography, and plain and contrast medium-enhanced digital radiography in studying root canal morphology. J Endod 2010 Sep;36(9):1547-1551.

3. Parekh V, Shah N, Joshi H. Root canal morphology and variations of mandibular premolars by clearing technique: an in vitro study. J Contemp Dent Pract 2011 Jul-Aug;12(4): 318-321.

4. Robertson D, Leeb IJ, McKee M, Brewer E. A clearing technique for the study of root canal systems. J Endod 1980 Jan;6(1):421-424.

5. Venturi M, Prati C, Capelli G, Falconi M, Breschi L. A preliminary analysis of the morphology of lateral canals after root canal filling using a tooth-clearing technique. Int Endod J 2003 Jan;36(1):54-63.

6. Verma M. Clearing Technique and its Potential ApplicationsAn in Vitro Study. J Indian Dent Assoc 2011;5(11):1122.

7. Mühlreiter E. Mühlreiter's Anatomie des menschlichen Gebisses: A. Felix 1920.

8. Black GV. Descriptive anatomy of the human teeth: The SS White manufacturing co; 1902.

9. Hess W, Zürcher E, Dolamore WH. The anatomy of the rootcanals of the teeth of the permanent dentition: William Wood; 1925.

10. Weine FS, Smulson MH, Herschman JB. Endodontic therapy: Mosby; 1972.

11. Okumura T. Anatomy of root canals: Tokyo Dental College; 1927.

12. Callis G, Sterchi D. Decalcification of bone: Literature review and practical study of various decalcifying agents. Methods, and their effects on bone histology. J Histotechnol 1998; 21(1):49-58.

13. Zillich R, Dowson J. Root canal morphology of mandibular first and second premolars. Oral Surg Oral Med Oral Pathol 1973;36(5):738-744.

14. Berutti E. Microleakage of human saliva through dentinal tubules exposed at the cervical level in teeth treated endodontically. J Endod 1996 Nov;22(11):579-582.

15. Tagger M, Katz A, Tamse A. Apical seal using the GPII method in straight canals compared with lateral condensation, with or without sealer. Oral Surg Oral Med Oral Pathol 1994 Aug; 78(2):225-231.

16. O'Neill KJ, Pitts DL, Harrington GW. Evaluation of the apical seal produced by the McSpadden compactor and the lateral condensation with a chloroform-softened primary cone. J Endod 1983 May;9(5):190-197.

17. Felton DA, Webb EL, Kanoy BE, Dugoni J. Threaded endodontic dowels: effect of post design on incidence of root fracture. J Prosthet Dent 1991 Feb;65(2):179-187.

18. Vertucci FJ. Root canal morphology of mandibular premolars. J Am Dent Assoc 1978 Jul;97(1):47-50. 